\title{
LATTICE SITES AND DAMAGE ANNEALING OF IMPLANTED Tm AND Er IN Si
}

\author{
U. Wahl ${ }^{1}$, J.G. Correia ${ }^{2}$, J. De Wachter ${ }^{1}$, G. Langouche ${ }^{1}$, J.G. Marques ${ }^{3}$, R. Moons ${ }^{1}$, \\ A. Vantomme ${ }^{1}$, and the ISOLDE collaboration ${ }^{2}$ \\ ${ }^{1}$ Instituut voor Kern- en Stralingsfysica, University of Leuven, Celestijnenlaan 200 D, \\ B-3001 Leuven, Belgium, email: ulrich.wahl@fys.kuleuven.ac.be \\ ${ }^{2}$ CERN-PPE, CH-1211 Genève 23, Switzerland \\ ${ }^{3}$ CFNUL, Av. Prof. Gama Pinto 2, P-1699 Lisboa Codex, Portugal
}

\section{ABSTRACT}

We have studied the lattice sites of Er in CZ Si single crystals by using conversion electron emission channeling from the isotope ${ }^{167 \mathrm{~m}} \mathrm{Er}(2.28 \mathrm{~s})$ which is the decay product of radioactive ${ }^{167} \mathrm{Tm}(9.25 \mathrm{~d})$. Following $60 \mathrm{keV}$ implantation of ${ }^{167} \mathrm{Tm}$ at a dose of $4 \times 10^{13} \mathrm{~cm}^{-2}$ and annealing at $600^{\circ} \mathrm{C}$, more than $90 \%$ of ${ }^{167 \mathrm{~m}} \mathrm{Er}$ is found close to tetrahedral insterstitial (T) sites. The tetrahedral fraction of ${ }^{167 \mathrm{~m}} \mathrm{Er}$ decreases considerably after $10 \mathrm{~min}$ annealing at $800^{\circ} \mathrm{C}$ and above. We attribute this to the onset of diffusion of the parent ${ }^{167} \mathrm{Tm}$ and its trapping at other defects, presumably oxygen atoms or clusters of $\mathrm{Tm} / \mathrm{Er}$.

\section{INTRODUCTION}

Ion implantation of rare earth elements into $\mathrm{Si}$ is known to result in the formation of luminescent centers and is considered as a possible means of fabricating Si-based optoelectronic devices [1]. A major problem with this approach, however, is the removal of implantation damage and its related non-radiative recombination centers. At the same time rare earth atoms should be incorporated into optically active sites and prevented from extensive diffusion, which leads to segregation into silicide phases [2]. In the case of Si:Er, light-emitting diodes operating at room temperature have already been demonstrated [2]. On the other hand, the microscopic structure of $\mathrm{Er}$ defects in $\mathrm{Si}$ is still a matter of debate [3-8]. This concerns in particular the lattice sites of $\mathrm{Er}$ and the possible role of oxygen as a ligand. Photoluminescence (PL) spectroscopy studies have identified a number of Er defects with different symmetry properties in Si [5]. The most intense PL yield resulted from two centers having cubic and axial symmetry. The cubic center occurred in both float-zone (FZ) and Czochralski (CZ) Si and was attributed to tetrahedral (T) interstitial Er, while the center with axial symmetry was only observed in $\mathrm{CZ} \mathrm{Si}$ and ascribed to Er-O complexes. Theoretical investigations also predict that $\mathrm{T}$ sites are the most stable sites for all oxidation states of isolated $\mathrm{Er}$ atoms in $\mathrm{Si}[6]$ but direct lattice location using the Rutherford backscattering (RBS) channeling technique only identified Er on substitutional [7] or hexagonal interstitial [8] sites. This discrepancy, however, is probably due to the fact that RBS requires Er implantation doses 50-100 times higher $\left(>10^{15} \mathrm{~cm}^{-2}\right)$ than the doses that are commonly used in producing good PL samples. A high concentration of Er is much more likely to form complexes or precipitates, though [3].

In this contribution we report on direct lattice location of $\mathrm{Er}$ in $\mathrm{CZ} \mathrm{Si}$ using the emission channeling technique [9], which makes use of the fact that charged particles emitted from radioactive isotopes in a single crystal experience channeling or blocking effects along crystallographic axes and planes. This leads to an anisotropic emission yield from the surface, which depends on the occupied lattice sites of the probe atoms. While emission channeling in itself is already a lowdose technique, position-sensitive detection further increases its efficiency so that only $5 \times 10^{10}$ 
probe atoms are needed for a complete lattice location measurement such as shown below in Fig.1.

\section{EXPERIMENT}

We used the radioactive isotope ${ }^{167} \mathrm{Tm}\left(\mathrm{t}_{1 / 2}=9.25 \mathrm{~d}\right)$ which decays into two excited states of ${ }^{167} \mathrm{Er}$ with half lives of $1.5 \mathrm{~ns}$ and $2.28 \mathrm{~s}$, respectively. While the $1.5 \mathrm{~ns}$ state emits L and M conversion electrons of 48 and $56 \mathrm{keV}$ energy, the decay of the $2.28 \mathrm{~s}{ }^{167 \mathrm{~m}} \mathrm{Er}$ isomeric state is accompanied by K, L and M electrons of 150, 199 and $206 \mathrm{keV}$. Both types of electrons can be used for lattice location purposes, and they probe the lattice sites of ${ }^{167} \mathrm{Er}$ during different time windows following the decay of ${ }^{167} \mathrm{Tm}$. Radioactive rare earth beams of mass 167 are available at the ISOLDE facility [10] at CERN. They consist mainly of the three neutron-poor isotopes ${ }^{167} \mathrm{Lu}$ $(52 \mathrm{~min}) \rightarrow{ }^{167} \mathrm{Yb}(17.5 \mathrm{~min}) \rightarrow{ }^{167} \mathrm{Tm}(9.25 \mathrm{~d})$ of the isobaric decay chain. Contributions from stable ${ }^{167} \mathrm{Er}$ in its ground state and neutron-rich isotopes are less than $10 \%$. Our samples were two $<100>$ oriented Czochralski (CZ) single crystals of $p$-Si:B $\left(5-14 \Omega \mathrm{cm}\right.$, implanted dose $4.4 \times 10^{13}$ $\left.\mathrm{cm}^{-2}\right)$ and $n$-Si:P $\left(1-10 \Omega \mathrm{cm}, 3.5 \times 10^{13} \mathrm{~cm}^{-2}\right)$. Following room temperature implantation of the above isotopes at $60 \mathrm{keV}$, samples were stored for a few hours until the short-lived isotopes had decayed into ${ }^{167} \mathrm{Tm}$. Annealing was done in vacuum better than $10^{-5} \mathrm{mbar}$. In order to measure the conversion electron emission yield as a function of the angle from different crystallographic directions we used a $30 \times 30 \mathrm{~mm}^{2}$ Si detector $(0.5 \mathrm{~mm}$ thick, $5 \mathrm{keV}$ FWHM energy resolution), consisting of $22 \times 22$ "pads" (or pixels), each of $1.3 \times 1.3 \mathrm{~mm}^{2}$, which was mounted at a distance of $285 \mathrm{~mm}$ from the sample. The 484 pads, coupled to VLSI preamplifier chips, were read out serially by a digital signal processor (DSP) and a sampling analog to digital converter (ADC). Such detector systems were recently developed at CERN in the context of high-energy physics collider experiments [11] and we have adapted them for use in electron emission channeling [12].

\section{RESULTS AND DISCUSSION}

The left hand side of Fig. 1 shows the normalized emission yield of conversion electrons from the ${ }^{167 \mathrm{~m}} \mathrm{Er} 2.28 \mathrm{~s}$ state, measured at room temperature after annealing the $n$-Si crystal at $600^{\circ} \mathrm{C}$ for $10 \mathrm{~min}$. Clearly visible are prominent channeling effects along axial $\langle 100\rangle$ and $\langle 111\rangle$ and planar $\{110\}$ directions, and less pronounced channeling effects along $\{100\}$ and $\{211\}$. On the contrary, the axial $\langle 110\rangle$ and planar $\{111\}$ and $\{311\}$ directions all show yields close to unity or below. The combination of these patterns provides direct evidence that the majority of ${ }^{167 \mathrm{~m}} \mathrm{Er}$ occupies sites close to the tetrahedral interstitial $(\mathrm{T})$ position. The $\mathrm{T}$ sites are perfectly aligned with $\langle 100\rangle,\langle 111\rangle,\{100\},\{110\}$ and $\{211\}$ lattice directions, leading to channeling of electrons emitted from these sites, but they are almost midway between $\langle 110\rangle$ atomic axes and $\{111\}$ and $\{311\}$ atomic planes, causing yield minima along these directions $[9,13]$. Note that due to the negative charge of the conversion electrons this is essentially the opposite behaviour to that observed for alpha emitter atoms on tetrahedral sites [14]. Major contributions from emitters on substitutional (S) sites can be ruled out, since these would show up as channeling effects along $\langle 110\rangle,\{111\}$ and $\{311\}$ directions. A comparable argument holds for hexagonal $(\mathrm{H})$ sites, which are located clearly off all $\{100\}$ atomic planes and are incompatible with the observed channeling along $\{100\}$.

Fig. 1:

Left column: channeling patterns from ${ }^{167 \mathrm{~m}} \mathrm{Er}(2.28 \mathrm{~s})$ following room temperature implantation of

${ }^{167} \mathrm{Tm}$ into $n$-Si CZ and annealing at $600^{\circ} \mathrm{C}$ for $10 \mathrm{~min}$. Shown are the normalized electron 


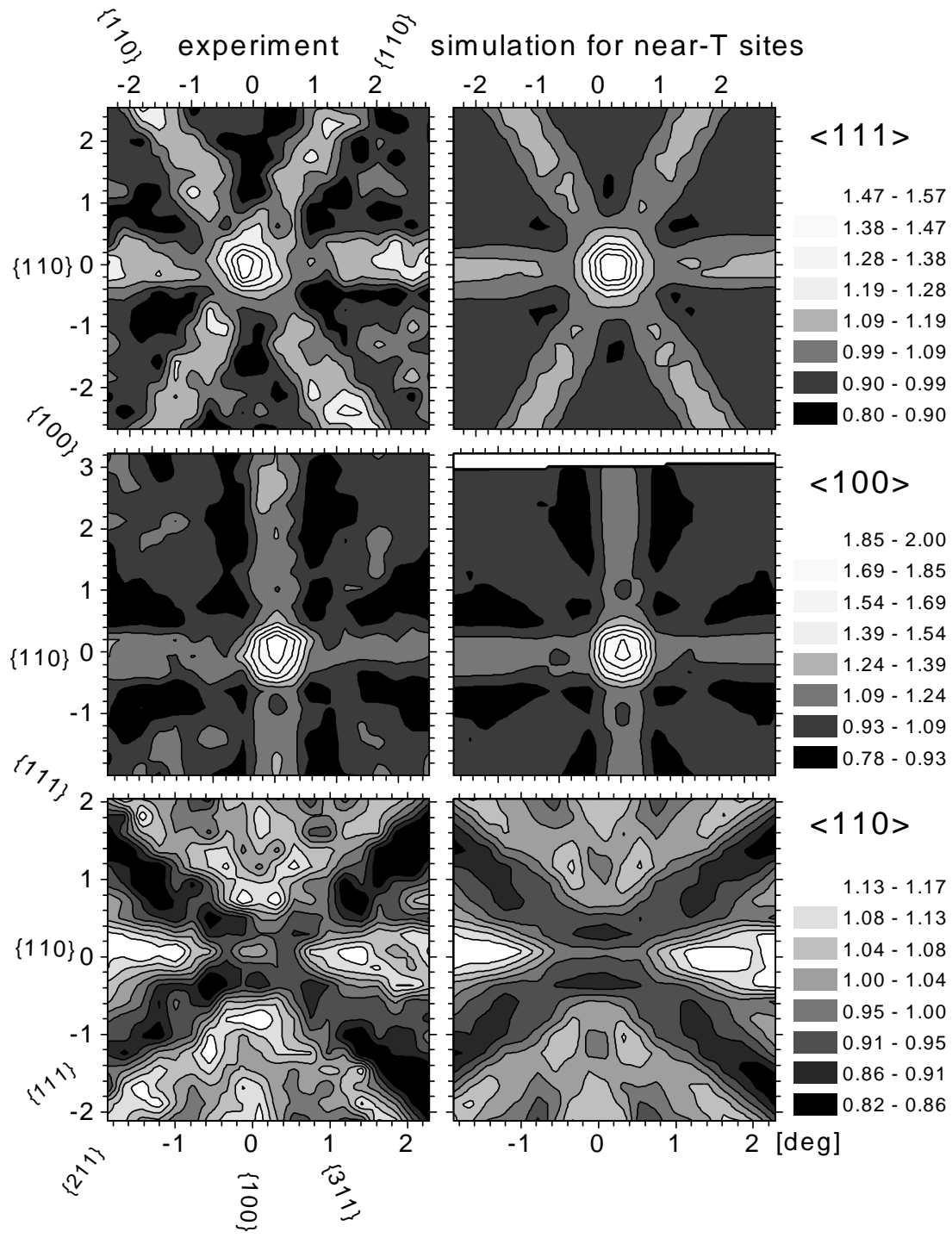

emission yields from the combined intensity of 150, 198 and 206 $\mathrm{keV}$ electrons around $\langle 111\rangle, \quad\langle 100\rangle$ and $<110\rangle$ directions.

Right column: best fits of simulated patterns to the experimental yields, corresponding to $92 \%, 88 \%$ and $96 \%$ of emitter atoms on sites which are displaced by $0.43 \AA$ from the $\mathrm{T}$ site. the lattice site precisely, we fitted the experimental patterns with theoretical emission yields from various lattice sites, which we calculated using the "many beam" approximation of the dynamical theory of electron diffraction. The concept of such computer simulations is described in detail in Refs. [9,13], and we have followed this approach with only one exception. Instead of using the approximation of plane waves, the dechanneling length for specific electron eigenstates due to incoherent scattering with lattice atoms was calculated by perturbation theory from the Bloch wave solutions, which gives a more accurate description of dechanneling [15]. A Debye temperature of $T_{\mathrm{D}}=504 \mathrm{~K}$ was used to calculate vibrational amplitudes of $\mathrm{Si}$ atoms [16], while thermal displacements of Er were estimated from an effective Debye temperature of $T_{\mathrm{D}}\left({ }^{167} \mathrm{Er}\right)=$ $(28.1 / 167)^{1 / 2} T_{\mathrm{D}}\left({ }^{28.1} \mathrm{Si}\right)=207 \mathrm{~K}$. Due to quantum-mechanical diffraction patterns, the angular dependence of the electron emission yield shows a rich fine structure, which requires us to regard a fine mesh of small angular steps. We therefore considered a range of $\pm 3^{\circ}$ around the $\langle 100\rangle$, $\langle 110\rangle$ and $\langle 111\rangle$ directions in steps of $\Delta x=\Delta y=0.05^{\circ}$, resulting in characteristic two-dimensional patterns of electron emission probability, $\chi_{\text {theo }}(\theta, \phi)$, where $\theta$ and $\phi$ denote polar and azimuth angles from the axis. The patterns for different electron energies were summed according to the conversion electron branching ratios, and smoothed using a Gaussian of $\sigma=0.06^{\circ}$ to account for that part of the experimental angular resolution which is due to the $1 \mathrm{~mm}$ beam spot on the 
sample. The size and shape of the detector pads was taken into account during fitting by averaging over the simulated yield falling within the angular range $\left(0.26^{\circ} \times 0.26^{\circ}\right)$ of one pad. We fitted theoretical emission patterns to the experimental yields $\chi_{\mathrm{ex}}$ according to

$\chi_{\text {ex }}(\theta, \phi)=S\left[f_{1} \chi_{\text {theo, } 1}(\theta, \phi)+f_{2} \chi_{\text {theo, },}(\theta, \phi)+1-f_{1}-f_{2}\right]$.

$S$ is a scaling factor common to all angles in one pattern, and $f_{1}$ and $f_{2}$ denote the fractions of emitter atoms on two different lattice sites. The so-called random fraction, $f_{R}=1-\left(f_{1}+f_{2}\right)$, accounts for emitter atoms which cause negligible anisotropies in emission yield, i.e. which are located on sites of very low crystal symmetry or in heavily damaged or amorphous surroundings. Up to six fitting parameters, $S, f_{1}, f_{2}, x_{0}, y_{0}, \phi_{0}$, were simultaneously optimized using non-linear least square fitting routines. Note that the parameter $S$ allows us to define the normalization of the experimental spectra, while $x_{0}, y_{0}$ and $\phi_{0}$ merely describe translational and azimuthal orientation with respect to the detector.

We considered S, T, H, bond center (BC), anti bonding (AB), split $\langle 100\rangle$ (SP) and the socalled $\mathrm{Y}$ and $\mathrm{C}$ sites, as well as $\langle 111\rangle$ and $\langle 100\rangle$ displacements between these sites (cf. Ref. [14] for site descriptions). Best fit results, however, were obtained (right hand side of Fig. 1) for Er atoms on lattice sites which are slightly displaced from the $\mathrm{T}$ site, and an additional small random fraction. The chi square of the fit showed comparable minima for 92(4)\% of Er on sites with static displacements of $d=0.43(8) \AA$ from either T to $\mathrm{H}, \mathrm{T}$ to $\mathrm{AB}$ or $\mathrm{T}$ to Y sites. Note that in the case of such small displacements the analysis of the channeling effect can only give mean values for the displacement of the emitter atoms. This means that it is not possible to determine whether this is due to static displacements of one specific value along all equivalent crystal axes, or an ensemble of Er atoms showing several small values of $d$ in the range $\approx 0-0.6 \AA$. We consider it very likely that we have a mixture of several lattice sites, with the majority of Er on ideal $\mathrm{T}$ sites and some smaller part on, e.g., nearby Y sites.

Fig. 2: Fraction of ${ }^{167} \mathrm{Er}$ on near-T sites obtained during isochronal annealing sequences (10 min, measurements at $20^{\circ} \mathrm{C}$ ).

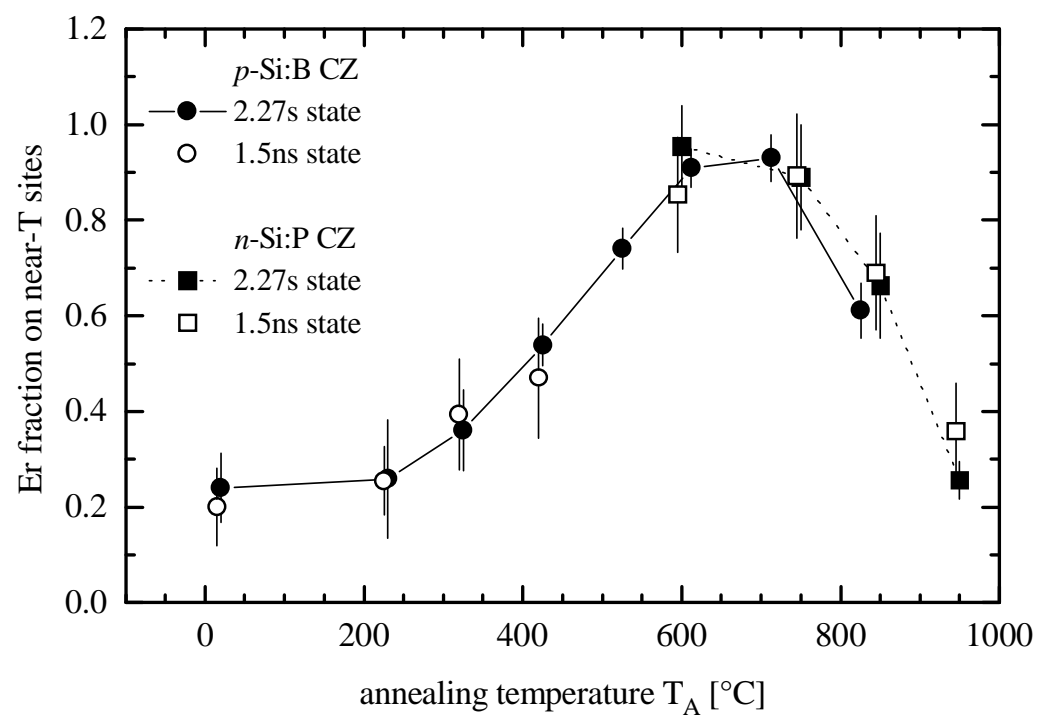

We also analyzed the emission channeling effects of the $48 \mathrm{keV}$ and $56 \mathrm{keV}$ electrons originating from the 1.5 ns state in ${ }^{167} \mathrm{Er}$ and obtained the same lattice preference as discussed above, i.e. 
near-T with $d=0.44(10) \AA$, with similar fractions (cf. Fig. 2). This shows that the majority of ${ }^{167} \mathrm{Er}$ already occupies the near-T sites within less than $1 \mathrm{~ns}$ after the ${ }^{167} \mathrm{Tm}$ decay.

Fig. 2 displays the Er fractions on near-T sites observed during isochronal annealing sequences of both $n$-Si and $p$-Si. Electron emission channeling effects from the $p$-type sample were already clearly visible directly following room temperature implantation. Fitting resulted in a near-tetrahedral fraction of $20-25 \%$ and the remainder on random sites. The fraction of probe atoms which are located in tetrahedral interstitial positions with only short-range order symmetry preserved, however, is probably even significantly higher. This is due to the fact that after implantation the ${ }^{167} \mathrm{Tm} /{ }^{167} \mathrm{Er}$ probes end up in a highly defective crystalline surrounding. Upon annealing to $600^{\circ} \mathrm{C}$ the electron emission channeling effects increased markedly. We attribute this to the well-known solid phase epitaxial regrowth of implanted $\mathrm{Si}$ in the temperature range 550$600^{\circ} \mathrm{C}$ (see, e.g., Ref. [17]). As a result, the quality of the crystal lattice is restored to a large extent, and our measurements show that subsequently more than $90 \%$ of ${ }^{167} \mathrm{Er}$ emitter atoms are located on near-T sites. For annealing above $750^{\circ} \mathrm{C}$ the fraction on near-T sites dropped markedly. The $n$-type sample was only investigated following annealing between $600^{\circ} \mathrm{C}$ and $950^{\circ} \mathrm{C}$, with essentially very similar results in the overlapping temperature region. The channeling patterns obtained after $950^{\circ} \mathrm{C}$ annealing of this sample could be well fitted by assuming $25-35 \%$ of Er on near-T sites and the remainder on random sites. However, within the statistical limits of our present data, we can not exclude small Er fractions $(<10 \%)$ on distinct lattice sites of low symmetry, e.g., $\mathrm{H}$ sites subsequent to these high-temperature annealing steps.

Let us discuss possible reasons for this decrease in the near-T Er fraction. The diffusivity of $\mathrm{Er}$ in $\mathrm{Si}$ is $\mathrm{D} \approx 10^{-15} \mathrm{~cm}^{2} \mathrm{~s}^{-1}$ at $900^{\circ} \mathrm{C}$ [2]. Assuming that the diffusivity of the parent $\mathrm{Tm}$ is similar to Er, one estimates a mean square diffusion length of $190 \AA$ during the 10 min annealing step. This is comparable to the average distance between oxygen atoms, which is of the order of magnitude of $130 \AA$ in $\mathrm{CZ} \mathrm{Si}$, but also comparable to the distance to neighbouring Er or Tm atoms. The latter value is $37 \AA$ in the peak of the implantation profile where the rare earth concentration reaches a maximum of $2 \times 10^{19} \mathrm{~cm}^{-3}$. Very recently we also carried out experiments in oxygen-lean FZ Si which showed that the stability of tetrahedral $\mathrm{Tm} / \mathrm{Er}$ is much higher in this material. This suggests that $\mathrm{O}$ is responsible for the decrease in the near- $\mathrm{T}$ fraction of ${ }^{167} \mathrm{Er}$ in $\mathrm{CZ} \mathrm{Si}$, acting as a precipitation center for diffusing rare earth atoms.

\section{CONCLUSIONS}

Our experiments provide the first direct evidence that Er can be incorporated on tetrahedral interstitial sites in $\mathrm{Si}$, as was already suggested by the existence of a cubic PL center [5]. Use of the emission channeling technique combined with position-sensitive detection has allowed these lattice location experiments while keeping the Tm/Er concentration below $2 \times 10^{19} \mathrm{~cm}^{-3}$. Compared to previous RBS studies of Si:Er [7,8], this represents 20-60 times lower implanted doses, and 10 -20 times lower concentrations, much closer to quoted values $\left(\approx 1-3 \times 10^{18} \mathrm{~cm}^{-3}\right)$ for manufacturing good luminescent samples $[2,4,5]$. Our data also indicate displacements from ideal $\mathrm{T}$ sites, which, however, could also be due to the existence of several different Er lattice sites in the range $\approx 0-0.6 \AA$ from the $\mathrm{T}$ sites. The near-tetrahedral fraction of ${ }^{167} \mathrm{Er}$ in $\mathrm{CZ}$ Si decreases considerably after annealing at $800^{\circ} \mathrm{C}$ and above for $10 \mathrm{~min}$. We attribute this to the onset of diffusion of the parent ${ }^{167} \mathrm{Tm}$ and its trapping at other defects, probably oxygen atoms or clusters of $\mathrm{Tm} / \mathrm{Er}$. In order to clarify the role of oxygen in this high-temperature annealing regime, comparison with lattice location experiments in oxygen-lean FZ Si is needed. Since the conversion electron transitions in ${ }^{167} \mathrm{Er}$ result in stable Er, this probe atom also offers the opportunity to initially investi- 
gate the Er lattice sites by emission channeling followed by a characterization of the luminescence properties of the same samples. Such studies are under way.

\section{ACKNOWLEDGEMENTS}

A. Vantomme acknowledges support from the post-doctoral research program of the Fund for Scientific Research, Flanders (FWO). J.G. Correia and J.G. Marques acknowledge their postdoc grants funded by JNICT, Portugal.

\section{REFERENCES}

[1] S. S. Iyer and Y. H. Xie, Science 260, 40 (1993).

[2] F. Y. G. Ren, J. Michel, Q. Sun-Paduano, B. Zheng, H. Kitagawa, D. C. Jacobson, J. M. Poate and L. C. Kimerling, Mat. Res. Soc. Symp. Proc. 301, 87 (1993).

[3] D.L. Adler, D.C. Jacobson, D.J. Eaglesham, M.A. Marcus, J.L. Benton, J.M. Poate and P.H. Citrin, Appl. Phys. Lett. 61, 2181 (1993).

[4] A. Polman, G.N. van den Hoven, J.S. Custer, J.H. Shin, R. Serna and P.F.A. Alkemade, J. Appl. Phys. 77, 1256 (1995).

[5] H. Przybylinska, W. Jantsch, Y. Suprun-Belevitch, M. Stephikhova, L. Palmetshofer,

G. Hendorfer, A. Kozanecki, R. J. Wilson and B. J. Sealy, Phys. Rev. B 54, 2532 (1996).

[6] M. Needels, M. Schlüter and M. Lannoo, Phys. Rev. B 47, 15533 (1993).

[7] Y. S. Tang, Z. Jingping, K. C. Heasman and B. J. Sealy, Sol. State Comm. 72, 991 (1989).

[8] A. Kozanecki, J. Kaczanowski, R. Wilson and B. J. Sealy, Nucl. Instr. Meth. B 118, 709 (1996).

[9] H. Hofsäss and G. Lindner, Phys. Rep. 201, 123 (1991).

[10] E. Kugler, D. Fiander, B. Jonson, H. Haas, A. Przewloka, H.L. Ravn, D.J. Simon, K. Zimmer and the ISOLDE collaboration, Nucl. Instr. Meth. B 70, 41 (1992).

[11] P. Weilhammer, E. Nygård, W. Dulinski, A. Czermak, F. Djama, S. Gadomski, S. Roe, A. Rudge, F. Schopper and J. Strobel, CERN-PPE/96-57, and submitted to Nucl. Instr. Meth.

[12] U. Wahl, J.G. Correia, A. Czermak, J. De Wachter, W. Dulinski, P. Jalocha, G. Langouche, J.G. Marques, R. Moons, E. Nygård, A. Rudge, F. Schopper, A. Vantomme and P. Weilhammer, to be published.

[13] H. Hofsäss, Hyperfine Interactions 97, 247 (1996).

[14] U. Wahl, ISOLDE collaboration, Phys. Rep. 280, 145 (1997).

[15] J. U. Andersen, E. Bonderup, E. Lægsgaard, B. B. Marsh and A. H. Sørensen, , Nucl. Instr. Meth. 194, 209 (1982).

[16] L. V. Hau, E. Lægsgaard, and J. U. Andersen, Nucl. Instr. Meth. B 48, 244 (1990).

[17] E. Rimini, Ion Implantation: Basics to Device Fabrication, Kluwer, Boston, 1995, p 173 ff. 